\title{
Diabetic retinopathy and OCT angiography: clinical findings and future perspectives
}

\author{
Jose Mauricio Botto de Barros Garcia*, David Leonardo Cruvinel Isaac and Marcos Avila
}

\begin{abstract}
In diabetic retinopathy (DR), macular involvement can present as either macular edema or ischemia. Fluorescein angiography remains the gold standard in the evaluation of retinal vascular perfusion and diagnosis of macular ischemia. However, it is a costly, time-consuming technique, it requires venipuncture, and reports of anaphylaxis and death related to fluorescein injections have been documented, despite their rarity. Optical coherence tomography (OCT) provides a fast and non-invasive method to assess retinal structures at a microscopic level. OCT angiography permits the noninvasive study of retinal and choroid circulation via motion contrast imaging. Split-spectrum amplitude decorrelation angiography combined with OCT angiography has furthered the understanding of retinal and choroidal vascular diseases, allowing the evaluation of retinal microvasculature and identification of subsequent disorders, including DR. Previous studies using OCT angiography have demonstrated that it may demonstrate DR findings such as microaneurysms, arteriolar wall staining, retinal neovascularization, and intraretinal microvascular abnormalities. The purpose of this article is to describe and discuss different concepts regarding OCT angiography, as well as its role in the diagnosis of DR and maculopathy.
\end{abstract}

Keywords: Macula, Retina, Imaging, Optical coherence tomography

\section{Background}

Approximately 347 million people worldwide have diabetes mellitus (DM) [1]. The worldwide prevalence of DM is predicted to grow to 430 million patients by 2030 [2]. Diabetic retinopathy (DR), a diabetic microangiopathy, is characterized by microaneurysms (MAs), capillary nonperfusion, and ischemia within the retina [3-6]. It may cause several complications, such as diabetic macular edema (DME) and diabetic macular ischemia (DMI) [1, 7-11]. In particular, capillary nonperfusion impairs the nutrition of the neuroglial tissues in the retinal parenchyma, and the resultant hypoxia increases the expression of vascular endothelial growth factor (VEGF), which promotes both angiogenic responses and vascular permeability [12]. Diabetic maculopathy is caused by a

*Correspondence: zemauricio20@hotmail.com

Federal University of Goias, Av. Primeira Avenida, S/N, Rua 234, 38, Apto

1011, Setor Leste Universitario, Goiania, GO CEP 74605-020, Brazil combination of both VEGF-mediated factors and inflammatory mediators [13-15].

DME occurs when high glucose levels cause capillaropathy that damages the blood supply to the retina. A hallmark of this disease is alterations in the bloodretinal barrier that is characterized by pericyte loss and endothelial cell-cell junction breakdown [16]. Numerous treatments have been established to manage DME, such as focal or grid photocoagulation and antiangiogenic therapy, which have been recently shown to yield good results in the treatment of DME $[7,11,17]$.

Fluorescein angiography is broadly recognized as an important tool in the diagnosis and treatment of DR [15]. However, it requires venipuncture, and reports of anaphylaxis and death related to contrast injections, although rare, have been documented [18]. In addition, the technique is costly and time-consuming, requiring up to $10 \mathrm{~min}$ for framing acquisition [19-21]. Nevertheless, it remains the gold standard in the analysis of DR features. 
The development of optical coherence tomography (OCT) has revolutionized ophthalmology and provides a rapid and noninvasive method to assess retinal structures at the microscopic level [20]. Spectral domain OCT (SD OCT) has become an important tool with which to manage patients with DR [17].

OCT angiography permits the noninvasive imaging of retinal and choroidal circulation via motion contrast imaging $[21,22]$. This relatively novel imaging technique obtains high-resolution volumetric blood flow information and generates angiographic images in a matter of seconds $[5,6,21,22]$. OCT angiograms are resampled with OCT B-scans from the same area, simultaneously allowing the assessment of structure and blood flow [21, 23].

The high speed of SD OCT compared with imedomain OCT (TD OCT) has made the imaging of both the structure and blood flow possible [24, 25]. Several OCT-derived techniques have been successfully developed to image in vivo human eye microcirculation, such as phase-variance OCT (PV OCT), phase contrast OCT (PC OCT), and split-spectrum amplitude decorrelation angiography (SSADA), which will be discussed herein [24-26].

The purpose of this article is to describe and discuss different concepts regarding OCT angiography, as well as its role in the diagnosis of DR and maculopathy.

\section{OCT angiography: technology applied to retinal disorders}

Since it was first described to capture ophthalmic images in 1991, OCT has increasingly been used in clinical practice and ophthalmic research [27]. OCT angiography is a further step in OCT technology that allows microvascular assessment by detecting blood flow. Different OCT angiography platforms and segmentation algorithms have been described, with the AngioVue software of RTVue XR Avanti (Optovue, Fremont, CA) being the first commercially available OCT angiography system [21, 22, 24]. This device obtains volumetric scans of $304 \times 304$ A-scans at 70,000 A-scans per second in approximately $3.0 \mathrm{~s}$. The $3 \times 3-\mathrm{mm}$ OCT angiograms appear to provide higher resolution images than the currently available imaging techniques [20]. SSADA aims to improve the signal to noise ratio (SNR) [22, 24, 28, 29]. Its application with OCT angiography instrumentation has furthered the understanding of retinal and choroidal vascular diseases.

Using SSADA, Spaide and co-workers demonstrated in vivo a distinct superficial capillary plexus (SCP) and deep capillary plexus (DCP), the latter of which includes the intermediate plexus $[28,30]$. Thus, OCT angiography could be used to enrich the understanding of ischemic conditions that may affect the different layers of retinal microcirculation, such as cotton wool spots, acute macular neuroretinopathy (AMN), paracentral acute middle maculopathy (DCP ischemia), and macular telangiectasia (MacTel) type 2 [28, 31-33]. Reports applying swept-source OCT technology (SS OCT) in patients with MacTel 2 have already shown structural abnormalities in the inner retina, such as retinal cavitation with draping of the internal limiting membrane and abnormalities in the outer retina, including disruptions of the photoreceptor ellipsoid segment that were not previously appreciated by fluorescein angiography [33].

The AngioPlex OCT angiography instrument (Carl Zeiss Meditec, Dublin, CA) improved the CIRRUS HD OCT scanning rate to $68,000 \mathrm{~A}$-scans per second and introduced a tracking software known as FastTrac retinal-tracking technology. The standard scanning patterns available include $3 \times 3$ and $6 \times 6 \mathrm{~mm}$ OCT angiograms. The angiographic images are generated using a complex algorithm that analyzes differences in both the intensity and phase information from repeated B-scans in the same position. This process is repeated at multiple adjacent positions to generate an en face flow volume. This algorithm is known as OCT microangiography-complex (OMAG) [34]. AngioPlex and AngioVue have been compared in clinical practice, and it was demonstrated that AngioPlex requires a shorter time and provides a higher number of images available for analysis with fewer motion artifacts [35]. Another SD OCT angiography device is the NIDEK RS-3000 (NIDEK, Aichi, Japan), which provides scans that range from $3 \times 3$ to $9 \times 9 \mathrm{~mm}$. Moreover, up to a $12 \times 9-\mathrm{mm}$ panorama image can be automatically generated.

SS OCT technology uses longer wavelength infrared light $(1050 \mathrm{~nm})$ than conventional SD OCT. This technology enables improved penetration into tissue and imaging through optical opacities and is invisible to the subject. The DRI OCT (Triton SS OCT; Topcon, Tokyo, Japan) imaging systems, including the Atlantis prototype technology, can acquire 100,000 A-scans per second in both healthy and diseased eyes. The volumetric OCT scans can be acquired over a $3 \times 3-\mathrm{mm}$ field of view in approximately $4 \mathrm{~s}$ of the total OCT scan time. Each B-scan position is repeatedly scanned 4 times. The examination field can be enlarged to $6 \times 6 \mathrm{~mm}$ (Triton SS OCT and Atlantis) or even to $12 \times 9 \mathrm{~mm}$ (Atlantis prototype only) [36]. The algorithm used is called OCTA Ratio Analysis (OCTARA). It is based on a ratio calculation in which the full spectrum is kept intact; therefore, the axial resolution is preserved. This method provides advantages over differentiation-based approaches while possessing improved sensitivity over methods based on amplitude 
decorrelation [36]. Stanga et al. studied diabetic maculopathy and PDR with the Triton SS OCT platform. The authors concluded that it provides additional information regarding the localization and morphology of $\mathrm{NV}$ in the optic disc (NVD) and in more than half of the NV elsewhere (NVE), suggesting that it is a noninferior technique to study posterior pole alterations compared with fluorescein angiography [37].

OCT angiography can assess retinal and choroidal microcirculation, identifying subsequent anatomical findings in many retinal diseases, including DR $[5,6,15$, $25,30,32]$. The technique allows the study of retinal deep plexi, while conventional fluorescein angiography only shows the superficial plexus. Thus, OCT angiography has been used to help diagnose DR-associated complications, but further reports are needed to expand our understanding of DR pathogenesis.

\section{Diabetic retinopathy $\mathrm{R}$ and $\mathrm{OCT}$ angiography}

Numerous clinical findings related to DR have been identified using OCT angiography [6, 20,38,39]. Structural OCT angiograms identified different lesions in singular stages of DR [6]. OCT angiography is not dependent on contrast injection, which helps to provide detailed information regarding capillaries without fluorescein leakage $[5,20]$.

Ishibazawa et al. [6] concluded that OCT angiographic techniques could be used to study the origin of microaneurysms, describing them as demarcated saccular or fusiform shapes of focally dilated capillary vessels in the inner retina (Fig. 1). Despite this improved identification, reports have demonstrated that the number of microaneurysms was significantly lower than that obtained by conventional angiography but also that OCT angiography had the added benefit of localizing these lesions to
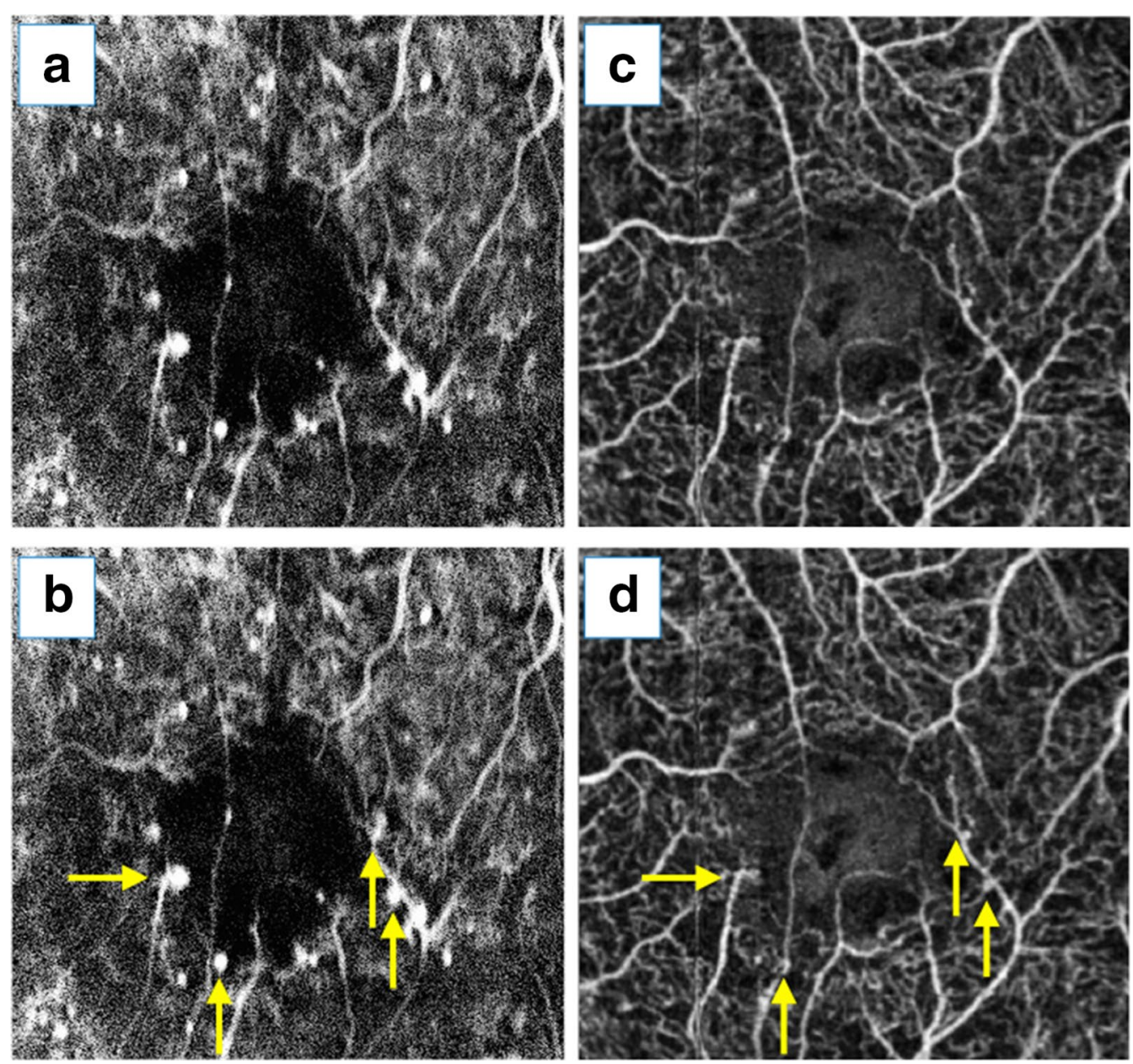

Fig. 1 Microaneurysm assessment using OCT angiography. This image was obtained from a diabetic patient and demonstrates microaneurysms. He was subjected to fluorescein angiography $(\mathbf{a}, \mathbf{b})$ and OCT angiography (c, d). Microaneurysm topography was compared using conventional angiography $(\mathbf{b})$ and a $3 \times 3-\mathrm{mm} \mathrm{OCT} \mathrm{angiogram} \mathrm{obtained} \mathrm{from} \mathrm{SCP} \mathrm{(d)} \mathrm{(yellow} \mathrm{arrows)}$ 
their exact intraretinal depths [13, 39]. Miwa et al. [12], using OCT angiography, detected only $41.0 \pm 16.1 \%$ of the microaneurysms identified in the fluorescein angiography images. Using $6 \times 6$-mm OCT angiograms, some authors were unable to reliably identify microaneurysms, probably due to their relatively low flow and scan concentrations [5]. Another possible reason for this result may be the focal staining of the capillary walls or surrounding tissues in the microaneurysms that clearly identifies them in the fluorescein angiography images, despite OCT angiography, because these factors are independent of erythrocyte movement [12].

Other DR clinical findings, such as arteriolar wall staining, neovascularization, and intraretinal microvascular abnormalities (IRMA), have divergent appearances on OCT angiography and fluorescein angiography. Wall staining and arteriolar narrowing have been illustrated as intense attenuation of microcirculation caliber on OCT angiography. IRMA, depicted as dilated terminal vessels surrounded by capillary loss, were similarly identified with OCT angiography and fluorescein angiography (Fig. 2) [5]. OCT angiograms clearly visualized new vessels on the disc (NVD) that persisted as spiral, looped, and irregular structures after initial anti-VEGF therapy [6]. Retinal NV is mostly detected by observing the flow signal above the internal limiting membrane. Hyperfluorescent lesions on fluorescein angiography that appeared indistinguishable from an MA were identified as NV using OCT angiography. This information may help us to understand why some patients with PDR and vitreous hemorrhage do not have a definitive NV on fluorescein angiography, as long as this method does not always identify all NV [5].

\section{Diabetic macular ischemia on OCT angiography}

Diabetic macular ischemia (DMI) is associated with functional retinal damage, and its diagnosis predicts DR progression $[4,19,40,41]$. It is characterized by the enlargement and disruption of the foveal avascular zone (FAZ) and by retinal capillary loss in other noncontiguous areas of the macula (capillary dropout) (Fig. 2) [41]. It has been postulated that the selective loss of pericytes and thickening of the basement membrane in retinal capillaries occur due to the effects of chronic hyperglycemia, leading to capillary occlusion, one of the characteristics findings of DMI [14]. Sim et al. [15, 40] demonstrated that approximately $41 \%$ of patients with DR in a tertiary hospital setting had some degree of macular ischemia. DMI is usually associated with reduced visual acuity (VA) in the eyes with moderate to severe grades of ischemia, but VA is preserved in milder grades of ischemia [10, 41, 42].
Because antiangiogenic therapy has become one of the treatments of choice for managing DR, the diagnosis of DMI has become more important, especially because antiangiogenic therapy can obscure the clinical findings related to ischemia progression [4, 19, 40, 41, 43]. Angiogenic factors, mainly VEGF, are believed to play a protective role in ischemic retinal disorders, improving volumetric blood flow and protecting retinal cells against apoptotic conditions [43]. Nevertheless, Campochiaro et al. [44] concluded that monthly injections of ranibizumab (Lucentis; Genentech, San Francisco, US) could diminish, but not completely prevent, retinal capillary closure in patients with DME.

Fluorescein angiography was introduced in 1961, and it has become the gold standard imaging modality for assessing the macular capillary network and its pathological conditions, such as macular ischemia [30, 43]. However, this method cannot distinguish between superficial and deep capillary plexi, as it displays in two-dimensional images the capillary bed of the superficial vascular plexus (Fig. 3) [20, 22, 30, 45].

Recent publications have shown that OCT angiography clearly depicts nonperfusion in $\mathrm{DR}[5,6,12,20]$. It provides an objective automated study of macular capillary nonperfusion as a potential sign of central ischemia [4]. Using OCT angiography with SSADA in healthy participants $(\mathrm{n}=105)$, Wang et al. measured a mean FAZ of $0.35 \pm 0.12 \mathrm{~mm}^{2}$. In addition to DMI, a larger FAZ was found in subjects with shorter axial lengths, hyperopia, increased subfoveal choroidal thicknesses, thicker lenses, shallower anterior chamber depths, and other characteristics [45]. Bradley et al. [15] demonstrated moderate agreement between the DMI grading results for OCT angiography (AngioVue platform) and fluorescein angiography in 24 diabetic patients using standard ETDRS protocols.

Hwang et al. [5] studied patients with proliferative DR (PDR), identifying FAZ enlargement and irregularities, as well as areas of capillary dropout. Miwa et al. [12] demonstrated that FAZ areas in OCT angiograms in the superficial layer were smaller than those in the fluorescein angiography images and were correlated with each other, agreeing with the finding that OCT angiography images often delineate the vascular structures in the nonperfused areas shown by fluorescein angiography. Usually, retinal microcirculation imaging is improved on OCT angiography despite fluorescein angiography (Fig. 4).

The identification of DCP by OCT angiography plays an important role in the setting of DMI (Fig. 5). Experimental studies have shown that the DCP contributes to photoreceptor inner segment oxygen requirements 

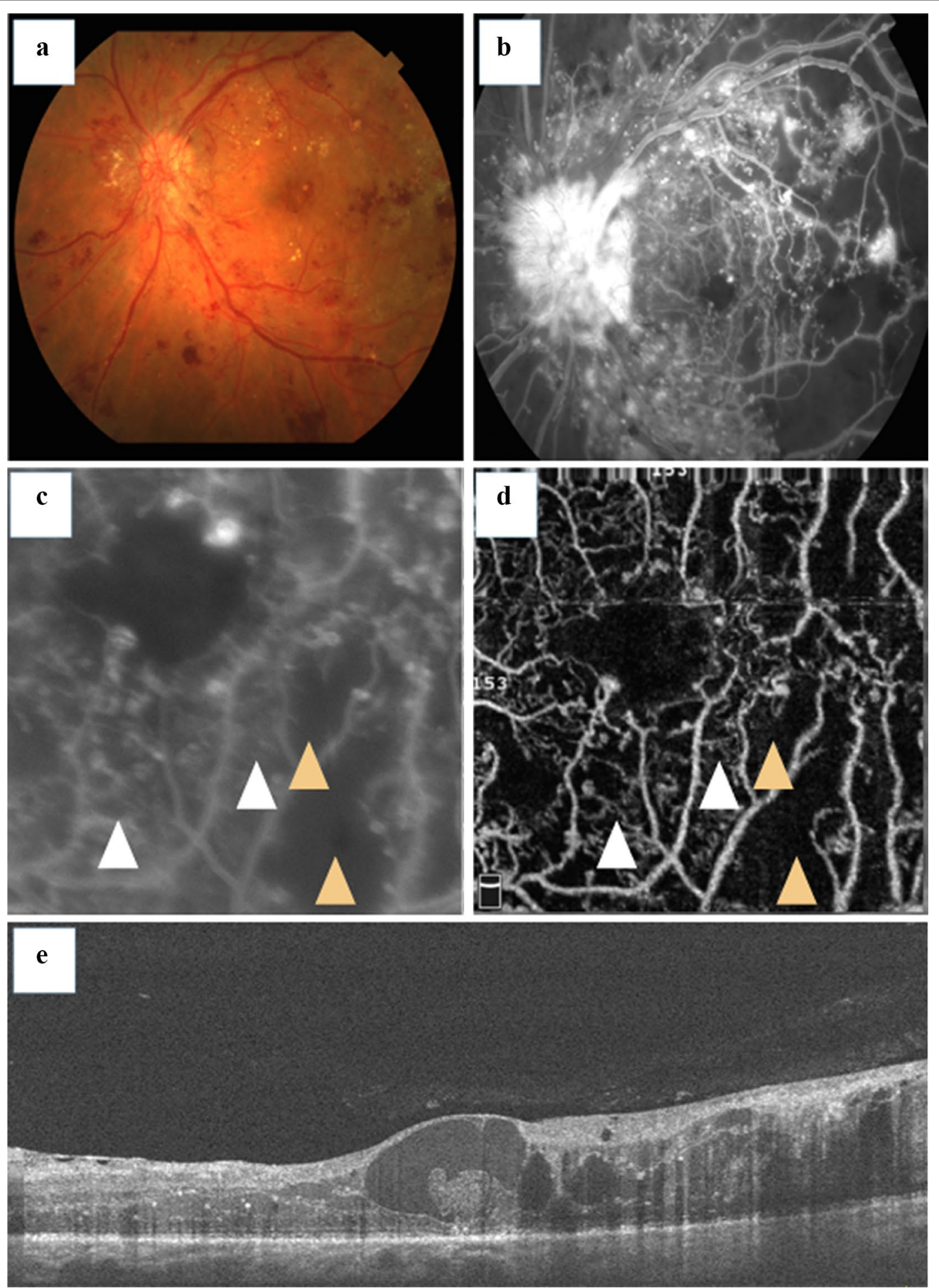

Fig. 2 Intraretinal microcirculation and capillary nonperfusion. This patient was diagnosed with PDR. Fundus photograph and fluorescein angiography $(\mathbf{a}, \mathbf{b})$. Images of $3 \times 3-\mathrm{mm}$ fluorescein angiography $(\mathbf{c})$ and OCT angiography were obtained $(\mathbf{d})$. The white arrowheads indicate superficial collaterals with adjacent nonperfusion areas (orange arrowheads) (c). The corresponding SD OCT B-scan is also shown (e) 

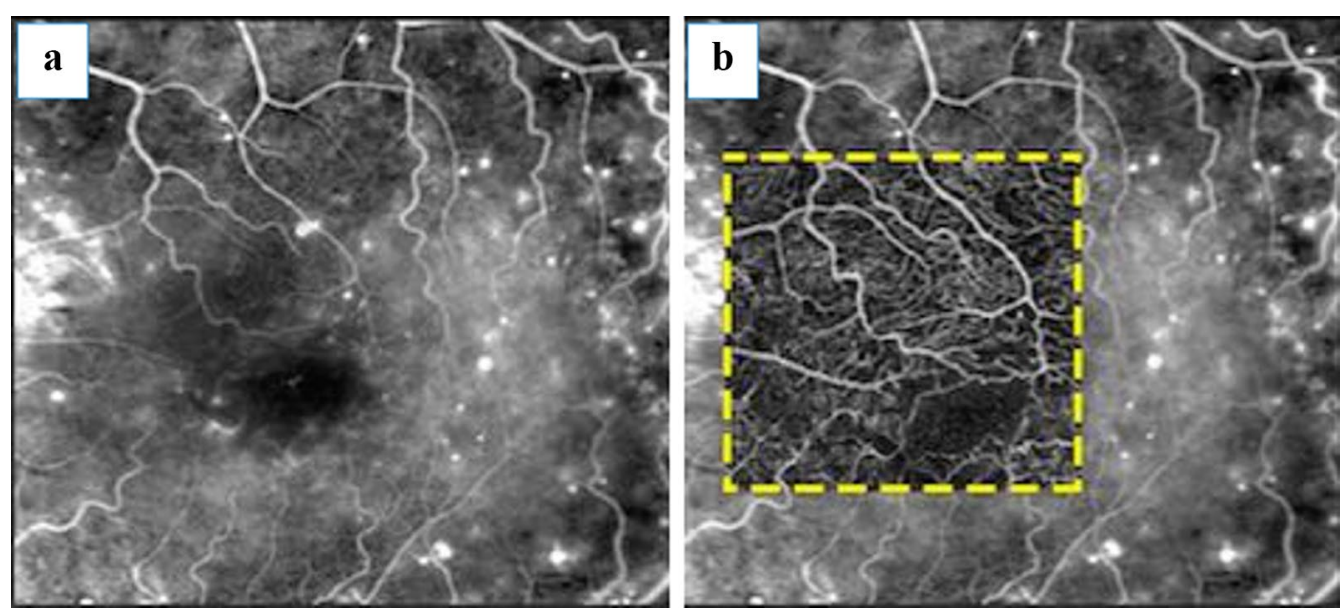

Fig. 3 Improving retinal imaging in DR on OCT angiography. This fluorescein angiography image (a) is overlapped by a $3 \times 3-\mathrm{mm}$ OCT angiogram obtained from the SCP (b), displaying better details in the absence of dye staining and leakage
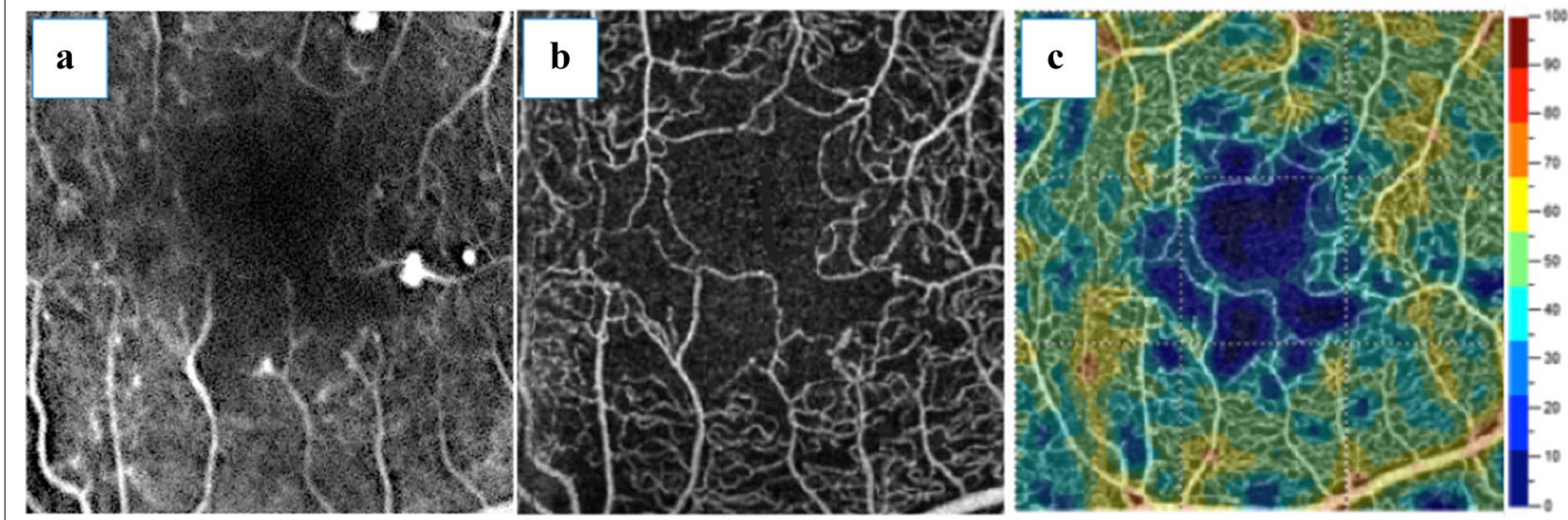

Fig. 4 Perifoveal perfusion density. Diabetic macular ischemia is demonstrated on fluorescein angiography (a) and a $3 \times 3-m m$ OCT angiogram obtained from the SCP $(\mathbf{b})$. Corresponding perfusion density mapping is represented by color-coded topographic maps with quantitative data (c)

(10-15\%), particularly during dark adaptation [46]. Recently, some authors have postulated that patients with DCP nonperfusion had an outer retina disruption on SD OCT in DMI, corresponding to areas of capillary nonperfusion at the level of the DCP [47, 48]. Yi et al. [49] demonstrated that during systemic hypoxia, the inner retina contribution to the metabolic needs of the outer retina microcirculation become even more significant because the choroidal vasculature fails to autoregulate its blood supply in the hypoxic setting. Couturier et al. [13] concluded that SCP capillary rarefaction was better identified on OCT angiography. Nevertheless, the DCP nonperfused areas were observed in only $35 \%$ of the subjects studied.

Recently, a novel quantitative graphic mapping technique for studying and displaying retinal vascular perfusion density using OCT angiography was developed. The authors applied software to images obtained from the Optovue SSADA algorithm that translates the OCT angiogram for each layer into qualitative and quantitative data using perfusion density analysis. Diabetic patients had significantly poorer capillary perfusion on SCP, DCP, and choriocapillaris than the controls in both the $3 \times 3$-mm and $6 \times 6$-mm settings. Subgroup analysis showed that normal subjects had higher capillary perfusion rates than patients diagnosed with mild non-proliferative DR (NPDR) (Fig. 6) [8].

\section{General perspectives and critical improvements}

Previously, the available OCT angiography devices were used to provide high-resolution OCT angiograms in patients with retinal diseases, including diabetic patients. 

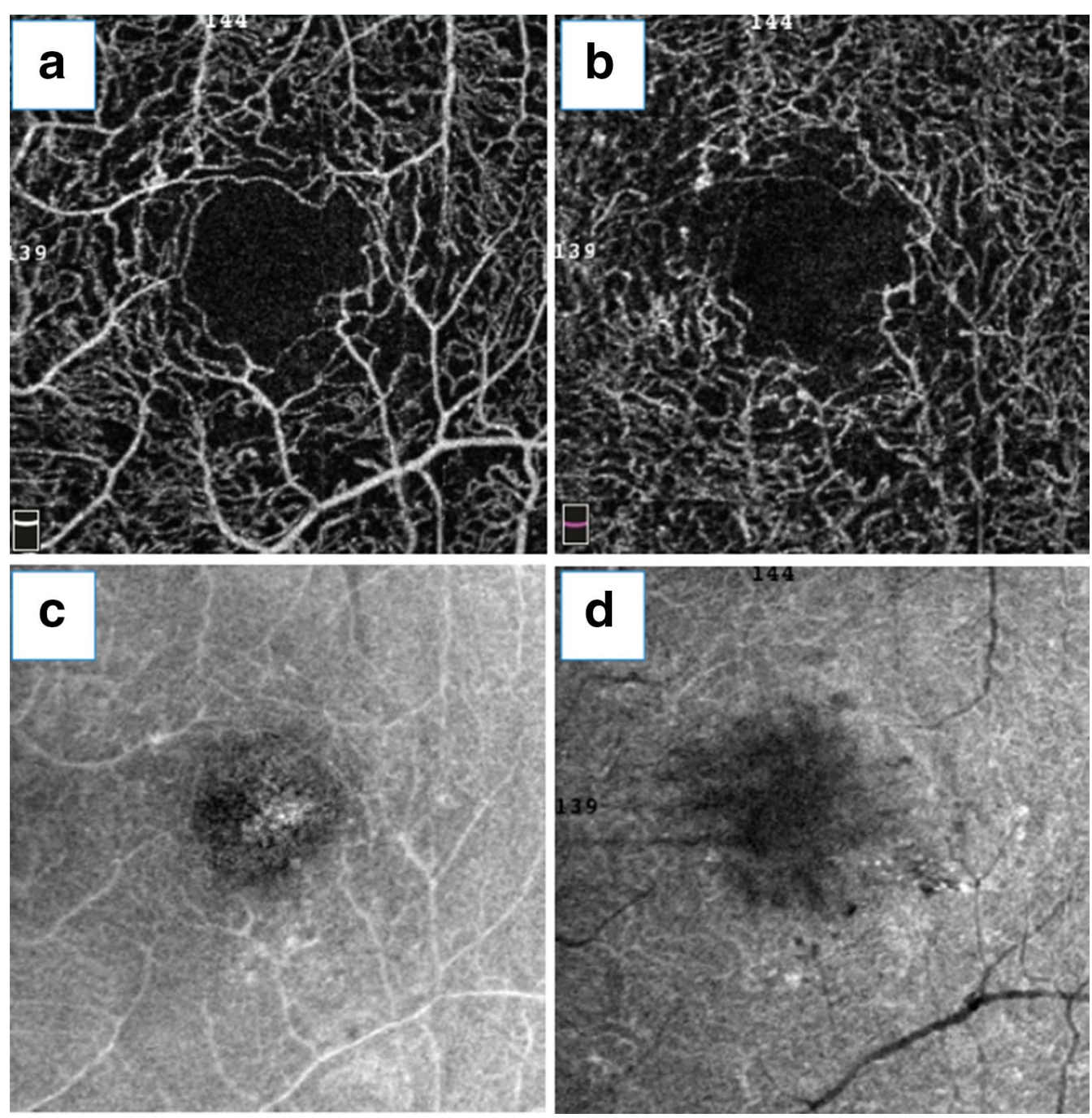

Fig. 5 Diabetic macular ischemia and OCT angiography. This patient was diagnosed with diabetic macular ischemia, represented by superficial capillary plexus (SCP) (a) and deep capillary plexus (DCP) (b) OCT angiography images. En face OCT angiograms from the SCP and DCP (c, d, respectively) are also displayed

Most of them apply the motion contrast concept to generate volumetric blood flow information, providing angiographic images after comparing sequential B-scans. However, other techniques have been described to image retinal blood flow, such as SS OCT, PV OCT, and Doppler OCT.

Doppler OCT has been used in conjunction with available SD OCT instrumentation. The Doppler effect is based on signals back-scattered from moving particles [24, 26, 50-52]. Doppler OCT is particularly useful for comparing depth scans, using this information to calculate the flow component parallel to the imaging direction (called axial flow) [51, 53]. Nevertheless, without dedicated scanning protocols, this technique is limited to the detection of strictly slow flow [54]. Furthermore, the inherent disadvantage of the angle dependence of the Doppler phenomenon has prevented it from becoming a reliable clinical tool for imaging retinal blood flow [52].

PV OCT has been used to image retinal circulation by Fingler et al. and Kim et al. The method differs from Doppler OCT by generating increased SD OCT imaging speeds, maintaining the previously demonstrated ability to display fast and slow blood flow independent of imaging orientation [51].

The SS OCT technique utilizes a $1050-n m$ wavelength instead of the $800-\mathrm{nm}$ wavelength used in most 

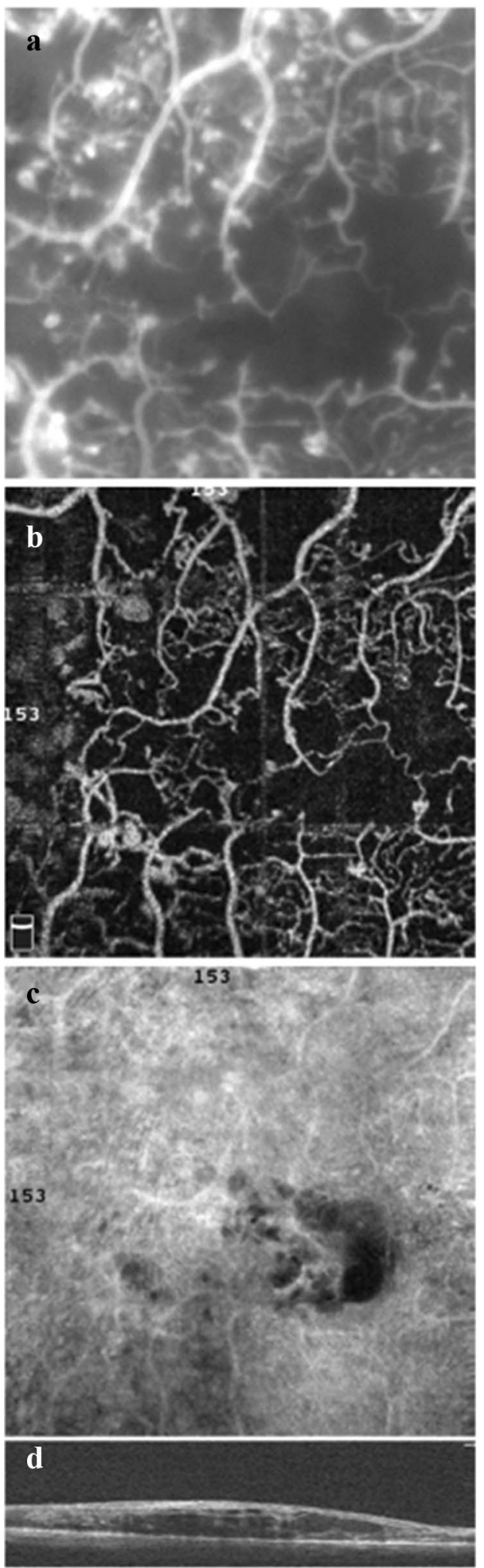

Fig. 6 Ischemic DME. Fluorescein angiography (a) compared with the $3 \times 3-\mathrm{mm} \mathrm{SCP} \mathrm{OCT} \mathrm{angiogram} \mathrm{(b).} \mathrm{DCP} \mathrm{(c)} \mathrm{and} \mathrm{corresponding}$ SD OCT B-scan (d) commercially available SD OCT instruments $[22,33,39$, 52]. There has been active research interest in exploring SS OCT systems at the $1-\mu \mathrm{m}$ wavelength range for imaging ocular posterior segments, offering the advantages of improved sensitivity roll-off and increased penetration depth into the choroidal tissue, higher resolution images, and a faster acquisition time than conventional SD OCT devices [52]. Thus, SS OCT angiography is a promising method that has numerous algorithms [33, 52]. The Massachusetts Institute of Technology has developed an OCT angiography prototype using this technology with a faster acquisition rate than that of commercially available SS OCT angiography instruments. This ultra-high-speed prototype employs a vertical cavity surface emitting laser (VCSEL) operating at a 1060-nm wavelength that allows increased light penetration into pigmented tissues and improved choroidal blood flow visualization compared with the light source used in SD OCT. This SS OCT angiography system obtains $500 \times 500 \mathrm{~A}$-scans at 400,000 A-scans per second in approximately $3.8 \mathrm{~s}$ [22].

Some commercial OCT angiography manufacturers are starting to include automated algorithms for mapping capillary density in their instrument software $[8,22]$. Because OCT angiography imaging is based on motion contrast, it is properly suited for assessing macula perfusion status, such as identifying regions with no detectable flow [8]. The follow-up image capture and intersession repeatability of automated vessel density measurements have been objectively demonstrated in healthy individuals. Further reports are needed in diabetic patients [55, 56]. In addition to mapping capillary perfusion, novel methods that allow the assessment of relative blood flow speeds have been developed. Variable interscan time analysis (VISTA), an OCT angiography imaging and analysis technique, was developed to overcome the limitation that typical OCT angiography does not directly measure blood flow speeds. Ploner et al. [57] concluded that abnormal flow speed was detected in several macular disorders, including DR.

OCT angiography must be properly compared with fluorescein angiography, as long as both methods have some limitations. OCT angiography requires that the patient fixate precisely for several seconds, whereas a useful fluorescein angiographic frame can be obtained in a fraction of a second [30]. In its current state, most commercially available OCT angiography systems suffer from motion artifacts and a relatively small field of view, which can be improved with further development efforts [4-6, 20, 25]. This instrument uses image alignment based on scanning laser ophthalmoscope (SLO) images obtained immediately prior to image capture. This eliminates the relatively 
long image acquisition time necessary for image capture when the automatic tracking function is used [56].

\section{Conclusions}

OCT angiography is a relatively new and promising imaging technique that uses SD OCT or SS OCT for the 3D visualization of the retinal and choroidal microcirculation without the need for dye injection. While fluorescein angiography is still considered the gold standard for imaging retinal vasculature in vivo, OCT angiography is a non-invasive, relatively fast imaging study that can be performed alongside routine OCT imaging. Several studies have demonstrated its applicability in diabetic patients, such as providing a prognosis and assessing treatment effects. Presently, OCT angiography allows the study of the retinal vascular bed and discriminates between superficial and deep retinal vascular plexi, which cannot be portrayed by conventional fluorescein angiography. In contrast, fluorescein angiography techniques are still superior in identifying slow blood flow structures such as microaneurysms. In summary, OCT angiography may provide images with greater detail regarding macular status and may become a novel imaging technique for the diagnosis of DMI in conjunction with fluorescein angiography in the management of DR.

\section{Authors' contributions}

JG performed the statistical analysis and drafted the manuscript. DI participated in the design and writing of the study, and MA participated in its design and coordination and helped to draft the manuscript. All authors read and approved the final manuscript.

\section{Competing interests}

The authors declare that they have no competing interests.

\section{Availability of data and supporting materials}

There are no data to be shared because the paper is a review of the clinical findings of diabetic retinopathy on OCT angiography. The included data were obtained from the literature and were supplemented with ideas from the authors regarding the main theme of this manuscript.

\section{Consent for publication}

This manuscript contains data from individual patients (including images), and these patients signed a consent form for their data to be published. No data from children were included in this manuscript.

\section{Ethics approval and consent to participate}

This was a retrospective cross-sectional comparative study analysis conducted at the Federal University of Goias and was approved by the ethics committee of the same institution. Signed informed consent was obtained from each subject prior to enrollment. This study was conducted in compliance with the tenets of the Declaration of Helsinki.

Received: 26 August 2016 Accepted: 22 February 2017

Published online: 13 March 2017

\section{References}

1. Varma R, Bressler NM, Doan QV, Gleeson M, Danese M, Bower JK, et al. Prevalence of and risk factors for diabetic macular edema in the United States. JAMA Ophthalmol. 2014;132(11):1334-40.
2. Korobelnik JF, Do DV, Schmidt-Erfurth U, Boyer DS, Holz FG, Heier JS, et al. Intravitreal aflibercept for diabetic macular edema. Ophthalmology. 2014;121(11):2247-54.

3. Adhi M, Brewer E, Waheed NK, Duker JS. Analysis of morphological features and vascular layers of choroid in diabetic retinopathy using spectral-domain optical coherence tomography. JAMA Ophthalmol. 2013;131(10):1267-74.

4. Hwang TS, Gao SS, Liu L, Lauer AK, Bailey ST, Flaxel CJ, et al. Automated quantification of capillary nonperfusion using optical coherence tomography angiography in diabetic retinopathy. JAMA Ophthalmol. 2016;134(4):367-73.

5. Hwang TS, Jia Y, Gao SS, Bailey ST, Lauer AK, Flaxel CJ, et al. Optical coherence tomography angiography features of diabetic retinopathy. Retina. 2015;35(11):2371-6.

6. Ishibazawa A, Nagaoka T, Takahashi A, Omae T, Tani T, Sogawa K, et al. Optical coherence tomography angiography in diabetic retinopathy: a prospective pilot study. Am J Ophthalmol. 2015;160(1):35-44.

7. Elman MJ, Aiello LP, Beck RW, Bressler NM, Bressler SB, Edwards AR, et al. Randomized trial evaluating ranibizumab plus prompt or deferred laser or triamcinolone plus prompt laser for diabetic macular edema. Ophthalmology. 2010;117(6):1064 e1035-1077 e1035.

8. Agemy SA, Scripsema NK, Chirag MS, Chui T, Garcia PM, Lee JG, et al. Retinal vascular perfusion density mapping using optical coherence tomography angiography in normals and diabetic retinopathy patients. Retina. 2015;35(11):2353-63.

9. Conrath J, Giorgi R, Raccah D, Ridings B. Foveal avascular zone in diabetic retinopathy: quantitative vs qualitative assessment. Eye (Lond). 2005;19(3):322-6.

10. ETDRS Research Group. Classification of diabetic retinopathy from fluorescein angiograms. Early treatment diabetic retinopathy study report number 11. Ophthalmology. 1991;98(5 Suppl):807-22.

11. ETDRS Research Group. Photocoagulation for diabetic macular edema. Early treatment diabetic retinopathy study report number 1. Arch Ophthalmol. 1985;103(12):1796-806.

12. Miwa Y, Murakami T, Suzuma K, et al. Relationship between functional and structural changes in diabetic vessels in optical coherence tomography angiography. Sci Rep. 2016;6:29064.

13. Couturier A, Mane V, Bonnin S, et al. Capillary plexus anomalies in diabetic retinopathy on optical coherence tomography angiography. Retina. 2015:35:2384-91.

14. Beltramo E, Porta M. Pericyte loss in diabetic retinopathy: mechanisms and consequences. Curr Med Chem. 2013;20(26):3218-25.

15. Bradley PD, Sim DA, Keane PA, et al. The evaluation of diabetic macular ischemia using optical coherence tomography angiography. Invest Ophthalmol Vis Sci. 2016;57(2):626-31.

16. 0

17. American Academy of Ophthalmology: American Academy of Ophthalmology Retina and Vitreous Panel. Preferred Practice Pattern ${ }^{\circledR}$ guidelines. Diabetic retinopathy. San Francisco, CA: American Academy of Ophthalmology; 2014. http://www.aao.org/ppp.

18. Yannuzzi LA, Rohrer K, Tindel $L$, et al. Fluorescein angiography complication survey. Ophthalmology. 1986;93(5):611-7.

19. Sim DA, Keane P, Fung S, et al. Quantitative analysis of diabetic macular ischemia using optical coherence tomography. Invest Ophthalmol Vis Sci. 2014;55(1):417-23.

20. Matsunaga D, Yi J, Puliafito CK, Kashani AH. OCT angiography in healthy human subjects. Ophthalmic Surg Lasers Imaging Retina. 2014;45(6):510-5.

21. de Carlo TE, Bonini Filho M, Chin AT, et al. Spectral-domain optical coherence tomography angiography of choroidal neovascularization. Ophthalmology. 2015;122(6):1228-38.

22. de Carlo TE, Romano A, Waheed NK, Duker JS. A review of optical coherence tomography angiography (OCTA). Int J Retina Vitr. 2015;1 (1):5.

23. Choi W, Mohler KJ, Potsaid B, et al. Choriocapillaris and choroidal microvasculature imaging with ultrahigh speed OCT angiography. PLOS ONE. 2013:8(12):e81499.

24. Jia Y, Tan O, Tokayer J, et al. Split-spectrum amplitude-decorrelation angiography with optical coherence tomography. Opt Express. 2012;20(4):4710-25 
25. Mastropasqua R, Di Antonio L, Di Staso S, et al. Optical coherence tomography angiography in retinal vascular diseases and choroidal neovascularization. J Ophthalmol. 2015;2015:1-8.

26. Gorczynska I, Migacz JV, Zawadzki RJ, Capps AG, Werner JS. Comparison of amplitude-decorrelation, speckle-variance and phase-variance OCT angiography methods for imaging the human retina and choroid. Biomed Opt Express. 2016;7(3):911-42.

27. Geitzenauer W, Hitzenberger C, Schmidt-Erfurth UM. Retinal optical coherence tomography: past, present and future perspectives. Br J Ophthalmol. 2011;95(2):171-7.

28. Nagiel A, Sadda S, Sarraf D. A promising future for optical coherence tomography angiography. JAMA Ophthalmol. 2015;133(6):629-30.

29. Tokayer J, Jia Y, Dhalla AH, Huang D. Blood flow velocity quantification using split-spectrum amplitude-decorrelation angiography with optical coherence tomography. Biomed Opt Express. 2013;4(10):1909-24.

30. Spaide RF, Klancnik JM Jr, Cooney MJ. Retinal vascular layers imaged by fluorescein angiography and optical coherence tomography angiography. JAMA Ophthalmol. 2015;133(1):45-50.

31. Rahimy E, Sarraf D, Dollin ML, Pitcher JD, Ho AC. Paracentral acute middle maculopathy in nonischemic central retinal vein occlusion. Am J Ophthalmol. 2014;158(2):372 e371-380 e371.

32. Spaide RF, Klancnik JM Jr, Cooney MJ. Retinal vascular layers in macular telangiectasia type 2 imaged by optical coherence tomographic angiography. JAMA Ophthalmol. 2015;133(1):66-73.

33. Thorell MR, Zhang Q, Huang Y, et al. Swept-source OCT angiography of macular telangiectasia type 2. Ophthalmic Surg Lasers Imaging Retina. 2014:45(5):369-80.

34. Rosenfeld PJ, Durbin MK, Roisman L, et al. ZEISS angioplex spectral domain optical coherence tomography angiography: technical aspects. Dev Ophthalmol. 2016;56:18-29.

35. De Vitis $L A$, Benatti $L$, Tomasso $L$, et al. Comparison of the performance of two different spectral-domain optical coherence tomography angiography devices in clinical practice. Ophthalmic Res. 2016;56(3):155-62.

36. Stanga PE, Tsamis E, Papayannis A, Stringa F, Cole T, Jalil A. Swept-source optical coherence tomography angio (Topcon Corp, Japan): technology review. Dev Ophthalmol. 2016;56:13-7.

37. Stanga PE, Papayannis A, Tsamis E, et al. New findings in diabetic maculopathy and proliferative disease by swept-source optical coherence tomography angiography. Dev Ophthalmol. 2016;56:113-21.

38. Huang D, Jia Y, Rispoli M, Tan O, Lumbroso B. Optical coherence tomography angiography of time course of choroidal neovascularization in response to anti-angiogenic treatment. Retina. 2015;35(11):2260-4.

39. Salz DA, de Carlo TE, Adhi M, et al. Select features of diabetic retinopathy on swept-source optical coherence tomographic angiography compared with fluorescein angiography and normal eyes. JAMA Ophthalmol. 2016;134(6):644-50.

40. Sim DA, Keane P, Zarranz-Ventura J, et al. Predictive factors for the progression of diabetic macular ischemia. Am J Ophthalmol. 2013;156(4):684-92.

41. Sim DA, Keane P, Zarranz-Ventura J, et al. The effects of macular ischemia on visual acuity in diabetic retinopathy. Invest Ophthalmol Vis Sci. 2013;54(3):2353-60.

42. ETDRS Research Group. Early photocoagulation for diabetic retinopathy. Ophthalmology. 1991;98(5 Suppl):766-85.

43. Manousaridis K, Talks J. Macular ischaemia: a contraindication for anti-VEGF treatment in retinal vascular disease? Br $\mathrm{J}$ Ophthalmol. 2012;96(2):179-84.
44. Campochiaro PA, Wykoff CC, Shapiro H, Rubio RG, Ehrlich JS. Neutralization of vascular endothelial growth factor slows progression of retinal nonperfusion in patients with diabetic macular edema. Ophthalmology. 2014;121(9):1783-9.

45. Wang Q, Chan S, Yang JY, et al. Vascular density in retina and choriocapillaris as measured by optical coherence tomography angiography. Am J Ophthalmol. 2016;168:95-109.

46. Birol G, Wang S, Budzynski E, Wangsa-Wirawan ND, Linsenmeier RA. Oxygen distribution and consumption in the macaque retina. Am J Physiol Heart Circ Physiol. 2007;293(3):1696-704.

47. Scarinci F, Nesper P, Fawzi AA. Deep retinal capillary non-perfusion is associated with photoreceptor disruption in diabetic macular ischemia. Am J Ophthalmol. 2016;168:129-38.

48. Scarinci F, Jampl L, Linsenmeier RA, Fawzi AA. Association of diabetic macular nonperfusion with outer retinal disruption on optical coherence tomography. JAMA Ophthalmol. 2015;133(9):1036-44.

49. Yi J, Liu W, Chen S, et al. Visible light optical coherence tomography measures retinal oxygen metabolic response to systemic oxygenation. Light Sci Appl. 2015;4(9):e334.

50. Tan O, Wang Y, Konduru RK, Zhang X, Sadda SR, Huang D. Doppler optical coherence tomography of retinal circulation. J Vis Exp. 2012;67:e3524.

51. Schwartz DM, Fingler J, Kim DY, et al. Phase-variance optical coherence tomography: a technique for noninvasive angiography. Ophthalmology. 2014;121(1):180-7.

52. Huang Y, Zhang Q, Thorell MR, et al. Swept-source OCT angiography of the retinal vasculature using intensity differentiation-based optical microangiography algorithms. Ophthalmic Surg Lasers Imaging Retina. 2014;45(5):382-9.

53. White $B$, Pierce $M$, Nassif $N$, et al. In vivo dynamic human retinal blood flow imaging using ultra-high-speed spectral domain optical coherence tomography. Opt Express. 2003;1 1(25):3490-7.

54. Grulkowski I, Gorczynska I, Szkulmowski M. Scanning protocols dedicated to smart velocity ranging in spectral OCT. Opt Express. 2009; 17(26):23736-54.

55. Al-Sheikh M, Tepelus TC, Nazikyan T, Sadda SR. Repeatability of automated vessel density measurements using optical coherence tomography angiography. Br J Ophthalmol. 2016. doi:10.1136/bjophthalmol-2016-308764 [Epub ahead of print].

56. Kita Y, Hollomicron G, Kita R, Horie D, Inoue M, Hirakata A. Differences of intrasession reproducibility of circumpapillary total retinal thickness and circumpapillary retinal nerve fiber layer thickness measurements made with the RS-3000 optical coherence tomograph. PLoS ONE. 2015;10(12):e0144721.

57. Ploner SB, Moult E, Choi W, et al. Toward quantitative optical coherence tomography angiography: visualizing blood flow speeds in ocular pathology using variable interscan time analysis. Retina. 2016;36:S118-26.

\section{Submit your next manuscript to BioMed Central and we will help you at every step:}

- We accept pre-submission inquiries

- Our selector tool helps you to find the most relevant journal

- We provide round the clock customer support

- Convenient online submission

- Thorough peer review

- Inclusion in PubMed and all major indexing services

- Maximum visibility for your research

Submit your manuscript at www.biomedcentral.com/submit
(O) BioMed Central 